\title{
Evidence for frames of reference based on pursuit eye movements
}

\author{
LEONARD D. STERN and DAVID EMELITY \\ University of Oregon, Eugene, Oregon 97403
}

\begin{abstract}
Lighted points that moved as if located on the rim of a rolling wheel were displayed to subjects whose task was to describe the pattern they perceived. The perceived patterns could be classified into one of four categories ranging from cycloidal to circular motion. Pursuit eye movements were controlled by having subjects track a fixation point that moved in the direction of the rolling wheel on a path just above the wheel's rim. With respect to the translatory velocity of the rolling wheel, the velocity of the fixation point was $100 \%$, $67 \%, 33 \%$, or $0 \%$ (i.e., stationary). The patterns traced out by the points on the wheel were perceived to become increasingly circular as pursuit eye movements more closely matched the translatory speed of the rolling wheel. This is taken to support Stoper's hypothesis that pursuit eye movements can establish a frame of reference for motion analysis.
\end{abstract}

When moving elements are observed, the frame of reference adopted by the observer can affect the motion that he perceives. For example, if a wheel with one illuminated point on its rim were rolled in a dark room, the lighted point would be perceived as describing a cycloidal path (see Figure 1a). However, if an additional light were placed on the hub of the wheel, the lighted point on the wheel's rim would be seen as tracing out a circular pattern about the horizontally moving light on the wheel's hub (Wallach, 1959). The common explanation for this phenomenon is that cycloidal motion is perceived when the observer's frame of reference is stationary; when a light is placed on the hub of the wheel, circular motion is perceived because the light on the wheel's hub serves as a frame of reference for the motion of the light on the wheel's rim.

How are frames of reference established? Two explanations have been offered. Johansson (1950) has proposed that when a number of items share components of motion, the common vector is automatically extracted by the visual system to act as the frame of reference for the remaining motion components. Hochberg and Fallon (1976) have recently found experimental evidence that verifies the role of this vector-abstraction process in establishing frames of reference. An alternative proposal has been made by Stoper (1973). Stoper suggests that a frame of reference can be established by the vector that is pursued by the eye; that is, in the case of the lighted points on the rolling wheel, when the eyes follow the

This project was supported in part by BRSG Grant RR 07080 awarded by the Biomedical Research Support Grant Program, Division of Research Resources, National Institutes of Health. The authors wish to thank Jacob Beck for his guidance and encouragement in this investigation. motion of the light at the wheel's hub, this point will act as the frame of reference around which the motion of a light on the rim will be perceived. According to Stoper's evidence, extraretinal signals from higher level centers to eye muscles, which control pursuit movements, are, unlike saccadic eye movements, not "taken into account" by the visual system in analyzing motion. Thus, the extraretinal signal and the signal derived from motion over the retina do not cancel each other out in pursuit eye movements. In the case of an eye pursuing the translatory motion of a moving wheel, this independence allows the translatory motion of the wheel to be carried by the extraretinal signal (and to act as a frame of reference) while the retinal signal carries the remaining circular motion.

The object of this study was to provide experimental evidence supporting Stoper's theory. Lighted points were displayed on a cathode ray tube (CRT) by a computer. The points behaved as if they were located on the rim of a rolling wheel. In addition to the points on the rim of the imaginary wheel, the computer displayed a fixation point just above the wheel's rim. This fixation point was capable of moving at various fixed speeds across the screen: its velocity ranged from zero up to the translatory velocity of the imaginary wheel. The prediction made in this study was that the pursuit eye movements used in tracking the fixation point would establish a frame of reference from which the motion of the lighted rim points would be perceived. Relative to the translatory motion of the imaginary wheel, the velocity of the fixation point was $100 \%, 67 \%, 33 \%$, or $0 \%$ (i.e., the fixation point remained stationary while the wheel rolled). The resulting motion of a single rim point that should have been perceived at each of these discrepancies between the fixation- 
point speed and the wheel speed was predicted mathematically from the following equations:

$$
\begin{gathered}
x=(v-V) t-[R \sin (w t)] \\
y=R[1-\cos (w t)] .
\end{gathered}
$$

Here, $v$ is the translatory velocity of the wheel, $\mathrm{V}$ is the horizontal velocity of the frame of reference (as determined by the velocity of the fixation point), $t$ is time, $R$ is the radius of the wheel, and $w$ is the angular velocity of the wheel. Figure 1 shows the patterns obtained for the four fixation-point-speed/ wheel-speed discrepancies used in this experiment.

A vector abstraction process could also bring about the predicted patterns of perceived motion described in Figure 1, since the motion of the fixation point could be extracted by the visual system and be used as a frame of reference for perceiving the motion of the points on the rim of the wheel. However, the crucial difference between the vectorabstraction and the pursuit-eye-movement hypotheses is that the pursuit-eye-movement account predicts that the perception of motion of the lighted rim points in the presence of a fixation point will be strongly affected by whether the eyes are pursuing the fixation point or not, whereas the vector-abstraction hypothesis predicts that there will be no effect of eye movements on perception. More specifically, the pursuit hypothesis predicts that even if a fixation point is traveling at $100 \%$ of the translatory velocity of the rolling wheel, when the eyes are held stationary, cycloidal (see Figure 1a) rather than circular motion will be perceived. The vector-abstraction hypothesis predicts that only the relative motion of the rim points and the fixation point is important in establishing a frame of reference, so the same motion will be per-

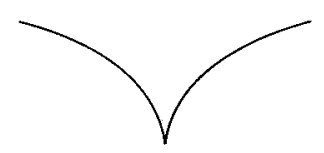

a

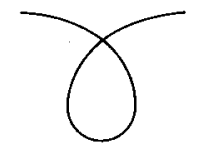

c

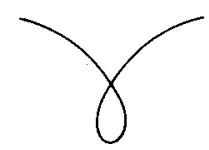

b

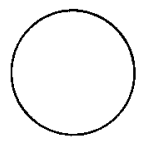

d
Figure 1. Patterns traced out by a single point on the rim of a rolling wheel when the rim point is viewed from a reference frame moving at $0 \%$ (a), $33 \%$ (b), $67 \%$ (c), or $100 \%$ (d) of the translatory velocity of the wheel. (Patterns a-d correspond to Responses $1-4$, respectively, made by the subjects.) ceived when the eyes are moving or stationary. Thus, if a fixation point is traveling at $100 \%$ of the translatory velocity of the rolling wheel, and if circular motion is perceived when the fixation point is pursued, the vector abstraction hypothesis predicts that circular motion will also be perceived when the eyes remain stationary.

To test the predictions made here, subjects in Experiments 1 and 2 viewed displays consisting of a single lighted point on the rim of a rolling wheel that was accompanied by a fixation point moving at either $100 \%, 67 \%, 33 \%$, or $0 \%$ of the translatory velocity of the rolling wheel. To determine whether the pattern of motion subjects perceived was, in fact, due to pursuit eye movements rather than to a vectorabstraction process, subjects fixated on a stationary point in the center of the display area and judged the motion of a point on the rim of the rolling wheel accompanied by a fixation point moving at $100 \%$ of the translatory velocity of the wheel.

In addition to displays which showed only a single lighted point on the imaginary wheel's rim, Experiment 1 also included displays in which there were between two and four lighted points on the wheel's rim. It was of interest to determine whether increasing the number of points having components of motion in common with that of the hub of the rolling wheel would, as predicted by a vector-extrapolation approach, influence perception.

\section{EXPERIMENT 1}

\section{Method}

Materials. A PDP-15 computer (manufactured by the Digital Equipment Corporation) displayed the stimuli and analyzed the data. All stimuli were shown on a CRT (Tektronix, Type 604) equipped with Type P11 phosphor, which has a decay rate of $20 \mathrm{msec}$ to $.1 \%$ of the original value. The CRT's display area measured approximately $9.2 \times 9.2 \mathrm{~cm}$ and was divided into a matrix of $512 \times 512$ points. The computer-refreshed points plotted on the CRT every $14 \mathrm{msec}$. Subjects used one of five keys to input their responses to the computer.

Visual display. If regarded as points on the rim of a rolling wheel, the display consisted of between one and four rim points separated by an angle of $90^{\circ}$. A fixation point, resembling the letter $X$, was programmed to appear $.7 \mathrm{~cm}$ above the rim of the rolling wheel. This fixation point measured $.6 \times .6 \mathrm{~cm}$. The points on the rim of the rolling wheel made 4 cycles across the screen; thus, the diameter of the imaginary wheel was $.73 \mathrm{~cm}$. The horizontal path of the hub of the wheel was $4 \mathrm{~cm}$ above the lowest edge of the CRT's display area. Subjects viewed the CRT's screen from a distance of $76 \mathrm{~cm}$; the CRT's display area, therefore, subtended an angle of $6.9^{\circ}$. A dim light in the experimental room made the borders of the screen visible.

The following sequence of events constituted a trial. For the $100 \%, 67 \%$, and $33 \%$ trials, the fixation point alone moved from left to right across the display area at what was to be the appropriate proportion of the translatory velocity of the wheel. As soon as the fixation point had disappeared from the screen, a rectangular grid pattern appeared at the center of the CRT's display area. This sequence of events enabled subjects to practice the eye movement necessary to pursue the fixation point that was to be dis- 
played in the presence of the to-be-judged rim-point pattern. Following a keypress and a delay of $1 \mathrm{sec}$, the fixation point moved across the screen again, at the same speed as in the practice run; however, when the fixation point had traveled a quarter of the distance across "the display area, the lighted point(s) on the rim of the moving wheel began the sweep across the screen. For the $100 \%$ trials, the fixation point reached the rightmost edge of the display area before the wheel-rim points; when this occurred, the entire display became blank so that the wheel-rim points were not being viewed in the absence of a fixation point. For the $100 \%$ trials, then, instead of the usual 4 cycles, the rim point(s) made only 3 cycles across the CRT's screen. For the $33 \%$ and $67 \%$ trials, the screen became blank after the fixation point (which was moving more slowly than the wheel) had traveled completely across the display area. A grid pattern then appeared in the center of the screen. Subjects used this as a cue to respond with a keypress. Following the keypress, the computer deleted the grid pattern and replaced it with the number of the response key (corresponding to Responses a, b, c, or d in Figure 1) that the subject had pressed; $500 \mathrm{msec}$ later, this number was deleted, and after a 1-sec interval the next trial began.

For trials in which the fixation point was to remain stationary (i.e., $0 \%$ trials), the fixation point was first displayed at a position halfway along its usual horizontal path. As in the other trials, once the subject depressed one of the response keys, the stationary fixation point was displayed again, this time accompanied by point(s) on the rim of a rolling wheel.

Trial definitions. Trial Block 1 presented a single lighted point on the rim of a rolling wheel accompanied by a fixation point that moved at $100 \%, 67 \%, 33 \%$, or $0 \%$ of the translatory velocity of the wheel. This block of trials will henceforth be referred to as the Single-Point Stimuli. The discrepancy between the velocity of the fixation point and that of the wheel as a whole was brought about by holding the speed of the wheel constant (at $14.6^{\circ} / \mathrm{sec}$ ) and varying the speed of the fixation point (Trials 1-4) and by holding the speed of the fixation point constant (at $11.6 \% / \mathrm{sec}$ ) and varying the speed of the wheel (Trials 5-7). In Trial 8, the speed of the wheel was $11.6^{\circ} / \mathrm{sec}$ and the fixation point remained stationary. Table 1 lists the Single-Point-Stimuli trial types.

Blocks 2 and 3 were used to display what will be termed here the Multipoint Stimuli-displays of between two and four points on the rim of the rolling wheel. The four points on the rim of the imaginary wheel were separated from each other by $90^{\circ}$. These points will be referred to as Points 1-4; according to this labeling scheme, then, Points 1 and 3 were separated from each other by $180^{\circ}$. In Block 2, the four discrepancies between the velocity of the rolling wheel and that of the fixation point were brought about by holding the speed of the rolling wheel constant (at $14.6^{\circ} / \mathrm{sec}$ ) and varying that of the fixation point. This was done for the patterns made up of Rim Points 1 and 2 (Set A), 1 and 3 (Set B), 1, 2, and 3 (Set C), and 1, 2, 3, and 4 (Set D)

Table 1

Single-Point Stimuli

\begin{tabular}{ccc}
\hline & \multicolumn{2}{c}{ Speed $^{\mathrm{a}}$} \\
\cline { 2 - 3 } Trial Number & Wheel & Fixation Point \\
\hline 1 & 14.6 & 14.6 \\
2 & 14.6 & 9.7 \\
3 & 14.6 & 4.8 \\
4 & 14.6 & Stationary \\
5 & 11.6 & 11.6 \\
6 & 17.4 & 11.6 \\
7 & 35.2 & 11.6 \\
8 & 11.6 & Stationary \\
\hline
\end{tabular}

measured in deg/sec at each of the four fixation-speed/wheel-speed discrepancies. For Block 3, the same fixation-speed/wheel-speed discrepancies were produced by having the speed of the fixation point remain constant (at $11.6 \% \mathrm{sec}$ ) while the speed of the rolling wheel varied. For the $0 \%$ trials in Block 3 , the fixation point was stationary and the wheel moved at $11.6^{\circ} / \mathrm{sec}$.

Block 4 displayed a single rim point and a fixation point moving at $100 \%$ of the translatory velocity of the imaginary wheel. The subjects viewed this display with their eyes held stationary on a "true" fixation point located in the center of the screen. The "dummy" fixation point and the wheel moved together at either $11.6^{\circ} / \mathrm{sec}$ (Trial 1) or $14.6^{\circ} / \mathrm{sec}$ (Trial 2). The Block 4 stimuli will be referred to as the Fixate-Stationary Stimuli.

Procedure. The subjects were told that they were going to see a number of lighted points moving across a screen and that these points would form patterns that resembled one of four prototype patterns. The subjects were shown the four patterns that are drawn in Figure 1. The four drawings were labeled with the numbers 1-4, as were a set of response keys. The subjects were instructed to observe each pattern that flashed across the screen and then to judge which of the four prototype patterns most closely matched what they had just seen. Response Keys 1-4 were used by the subjects to signal their judgments. Judgments $1-4$ corresponded to the patterns shown in Figures $1 \mathrm{a}, 1 \mathrm{~b}, 1 \mathrm{c}$, and $1 \mathrm{~d}$, respectively. If no judgment could be made, the subjects were told to press Response Key 5, the "error"' key.

Subjects were informed that they would be required to track a fixation point with their eyes. They were told that, to make their task a little easier, they would be given a practice run for each trial. This practice run consisted of a sweep of just the fixation point (or a display of just the fixation point in a stationary position) in the absence of any to-be-judged pattern.

After explaining the sequence of keypresses required in the experiment, and after answering any questions, the experimenter seated subjects before a CRT. A chinrest was used to keep each subject's head stationary and to position each subject's eyes $76 \mathrm{~cm}$ from the CRT's screen. The experimental room was illuminated by a dim light which permitted subjects to see the four prototype figures before them as well as the numbered response keys.

Each subject was given practice with the Single-Point Stimuli; four repetitions of each of the eight trial types were displayed for this purpose. The computer automatically randomized the sequence in which these trial types were presented. Following this practice session, the experimenter answered any additional questions the subjects had and then began the experiment. Each subject was shown eight repetitions of each Single-Point-Stimulus trial type (presented in a random order). After a brief rest, the subjects were shown two repetitions of each of the two Fixate-Stationary trials. For these trials, the experimenter affixed a small $(.3-\mathrm{cm}$ diam) white dot to the center of the CRT's screen to serve as the "true" fixation point. This fixation point was placed approximately $.8 \mathrm{~cm}$ above the path of the "dummy" fixation point. The subjects were told to fixate on the white dot; they were told the visual display would include both a moving fixation point and a moving pattern and that their task, as before, was to judge the form of this moving pattern. After these trials, the Multipoint Stimuli (Blocks 2 and 3) were displayed with four repetitions of each of the 16 trials in each block presented in a random order. Before being shown these last two blocks of stimuli, the subjects were told that the patterns they would see would be more complex than those that had been previously shown, but that the same four prototype patterns would be evident in the motions of the lighted points.

The experiment took approximately $\mathbf{4 5} \mathrm{min}$ for each subject to complete. The subjects were run individually and were free to set their own pace.

Subjects. Three paid subjects, recruited through the University of Oregon's Cognitive Laboratory subject pool, were used in this experiment. All subjects reported that they had no visual abnormalities. 


\section{EXPERIMENT 2}

The results and discussion of Experiment 1 will be delayed until Experiment 2 has been described. In Experiment 2, an attempt was made to monitor eye movements: while subjects viewed the Single-Point and Fixate-Stationary Stimuli, their eye movements were videotaped. The judgments given by the subjects in Experiment 2 were subsequently edited to exclude responses made on trials which, in the judgment of a second experimenter naive to the subjects' performances, were not accompanied by proper pursuit eye movements. This procedure was expected to improve the agreement between predicted and actual judgments given by subjects to the Single-Point Stimuli.

\section{Method}

Apparatus and Procedure. Except for the videotaping equipment, the apparatus for Experiment 2 was identical to that used in Experiment 1. The videotaping equipment present in the experiment room consisted of a portable camera mounted on a tripod at the level of the subjects' eyes and approximately $80 \mathrm{~cm}$ away from the subjects. The camera's zoom lens was adjusted so that the monitor's screen, located outside the experiment room, was completely filled with a close-up view of both eyes of each subject. The video equipment required the lighting in the experiment room to be brighter than it had been during Experiment 1.

To enable the experimenter to determine at what point in the recording the subjects were supposed to begin and end their eye movements, the computer switched on a tone generator for the duration of each sweep of the fixation point. This tone was recorded on tape together with the subjects' eye movements.

As in Experiment 1, the subjects were shown eight repetitions of each Single-Point-Stimulus trial type and two repetitions of each Fixate-Stationary trial type.

Subjects. Eight subjects, all female undergraduate students at the University of Oregon, participated in the experiment. Subjects received class credit for taking part in the experiment. Due to an equipment malfunction which prevented all responses from being recorded by the computer, the results of three of the subjects were lost. In addition, one subject reported that she was unable to perform the experimental task properly, so her data were discarded. Of the remaining four subjects, the data from one subject were not analyzed by the videotape procedure, since the glasses she was wearing caused a glare that obscured the view of her eye movements. All subjects reported they had normal or corrected-to-normal vision.

Data analysis. Videotapes of the three subjects were viewed once by the experimenter who had not been present when the subjects had performed the experiment. This experimenter judged whether the subjects had made appropriate pursuit eye movements for each $100 \%, 67 \%$, and $33 \%$ discrepancy trial. Either $a+$ or $a-$ was marked next to each trial number on the experimenter's sheet to indicate his decision. These judgments were then used to delete unsatisfactory trials for each of the three subjects. The absence of any pursuit eye movements, saccadic eye movements, or delays in commencing pursuit eye movements were used as bases for judging eye movements to be unsatisfactory.

\section{Results}

The judgments made by each subject, of the patterns traced out by the rim point(s), were coded as numbers 1-4; these numbers corresponded to the pat- terns shown in Figures $1 \mathrm{a}, 1 \mathrm{~b}, 1 \mathrm{c}$, and 1d, respectively. For the Single-Point Stimuli, all responses given by subjects in Experiment 2 (including those that would later be deleted using the videotape reviewing procedure) were combined with those given by subjects in Experiment 1. The average judgments for the Single-Point Stimuli are listed in Table 2. In this table, the two methods of achieving a discrepancy between fixation-point speed and the speed of the rolling wheel (i.e., keeping the wheel speed constant and changing the fixation-point speed, or keeping the fixation-point speed constant and changing the wheel speed) have been combined: An analysis of variance showed that there was no difference in the subjects' mean responses to the stimuli produced by these two methods of achieving a discrepancy $[\mathrm{F}(1,6)<1]$; furthermore, when the two methods of achieving discrepancies were compared at each of the four discrepancies between fixation-point speed and wheel speed, none of the $F$ values obtained were significant (all Fs $<2$ ).

To determine whether the Single-Point Stimuli were perceived as tracing out patterns that became more circular as the speed of the fixation point approached that of the translatory velocity of the rolling wheel, the judgments given to the Single-Point Stimuli, as a function of Fixation-Point-speed/wheelspeed discrepancy, were analyzed for a linear trend. The $F(1,6)$ value for the individual subjects' mean response data (see Table 2$)$ was $24.06(p<.01)$. A regression line for these data had the form $y=$ $.60 x+.83$. Thus, according to this equation, the average ratings given to the single-point patterns increased by .60 units for each of the four decreases in the discrepancies between the fixation-point and wheel speeds used in Experiments 1 and 2.

The Fixate-Stationary Stimuli were included in Experiments 1 and 2 to help differentiate between the effects of pursuit eye movements and a vectorextrapolation process. The relevant comparison here was between trials in which the fixation point and the wheel, both moving at the same velocity, were viewed with either the eyes tracking the fixation point or with the eyes held stationary. For Trials 1 and 5 of the Single-Point Stimuli, in which the eyes tracked a fixation point moving at $100 \%$ of the translatory

Table 2

Responses to the Single-Point Stimuli, Experiments 1 and 2 Combined

\begin{tabular}{ccccc}
\hline $\begin{array}{c}\text { Trial } \\
\text { Numbers }\end{array}$ & $\begin{array}{c}\text { Fixation Speed/ } \\
\text { Wheel Speed }\end{array}$ & $\begin{array}{c}\text { Predicted } \\
\text { Responses }\end{array}$ & $\begin{array}{c}\text { Mean } \\
\text { Responses }\end{array}$ & $\boldsymbol{\sigma}^{2}$ \\
\hline 1,5 & 1.00 & 4 & 3.24 & .86 \\
2,6 & .67 & 3 & 2.60 & .60 \\
3,7 & .33 & 2 & 2.03 & .65 \\
4,8 & 0.00 & 1 & 1.52 & .76 \\
\hline
\end{tabular}


FRAMES OF REFERENCE

velocity of the wheel, the mean judged response was 3.24. For the Fixate-Stationary Stimuli, the responses given when this same relationship between the moving fixation point and wheel was viewed with the eyes held stationary was 1.74 (Trials 1 and 2 combined; $\sigma^{2}=$ $.41)$. A correlated t test showed the difference between these two means was significant $[\mathrm{t}(6)=7.08$, $\mathrm{p}<.01]$.

Additional support for the eye-movement hypothesis was obtained from a mean error score computed for the three subjects in Experiment 2 whose eye movements were taped and analyzed. This error score was obtained by subtracting the actual from the predicted response on each $100 \%, 67 \%$, and $33 \%$ trial of the Single-Point Stimuli, squaring this difference and finding the square root of each subject's average squared difference score. According to an eye-movement account, excluding judgments based on improper pursuit eye movements should reduce the difference between actual and predicted responses; this should lead to smaller error scores. For the Single-Point Stimuli, an error score was computed for both the complete, unedited sets of responses of each of the three subjects, and for the sets of responses remaining when judgments based on unsatisfactory eye movements were removed. The average error score for the unedited responses was $.93\left(\sigma^{2}=.09\right)$. The editing process which required removing between 13 and 28 trials from each subject's 64 trials reduced the average error score to $.82\left(\sigma^{2}=.09\right)$. The difference between these means approached significance at the .05 level $[\mathrm{t}(2)=2.3$, $.05<\mathrm{p}<.1$ ]. A regression line for the edited data had a slope of .62 and an intercept of .77 .

In addition to investigating the role of eye movements in establishing frames of reference, the design used in Experiments 1 and 2 also permitted the role of a vector-extrapolation process to be assessed. A vector-extrapolation account of the process of establishing frames of reference proposes that the motion of the lowest velocity vector that a set of points has in common will ast as the frame of reference. Thus, for the Multipoint Stimuli used in Experiment 1, the vector-extrapolation hypothesis predicts that the more points there are on the rim of the wheel (points that share the motion of the wheel's hub), the more readily the motion of the rim points will be perceived as being circular. In terms of the response numbers used in these experiments, as more rim points were added to the display, the responses given to the patterns should have tended to become higher. Table 3 lists the combined data for the Multipoint Stimuli. This table shows the responses for the patterns formed by displaying between two and four lighted points on the rim of the wheel. For each of the combinations of points, a regression line was fitted to the four fixation-speed/wheel-speed discrepancy conditions. The functions resulting from
Table 3

Data for the Multipoint Stimuli

\begin{tabular}{lcccr}
\hline Points & $\begin{array}{c}\text { Fixation Speed/ } \\
\text { Wheel Speed }\end{array}$ & $\begin{array}{c}\text { Predicted } \\
\text { Responses }\end{array}$ & $\begin{array}{c}\text { Mean } \\
\text { Responses }\end{array}$ & \multicolumn{1}{c}{$\sigma^{2}$} \\
\hline 1,2 & 1.00 & 4 & 3.29 & 1.13 \\
1,2 & .67 & 3 & 2.67 & .57 \\
1,2 & .33 & 2 & 1.75 & .26 \\
1,2 & 0.00 & 1 & 1.42 & .36 \\
1,3 & 1.00 & 4 & 3.50 & .93 \\
1,3 & .67 & 3 & 3.04 & .83 \\
1,3 & .33 & 2 & 1.67 & .33 \\
1,3 & 0.00 & 1 & 1.17 & .17 \\
$1,2,3$ & 1.00 & 4 & 3.92 & .12 \\
$1,2,3$ & .67 & 3 & 3.29 & .66 \\
$1,2,3$ & .33 & 2 & 1.85 & .80 \\
$1,2,3$ & 0.00 & 1 & 1.54 & 1.35 \\
$1,2,3,4$ & 1.00 & 4 & 4.00 & 0.00 \\
$1,2,3,4$ & .67 & 3 & 3.79 & 2.0 \\
$1,2,3,4$ & .33 & 2 & 2.17 & .56 \\
$1,2,3,4$ & 0.00 & 1 & 1.82 & 2.17 \\
\hline
\end{tabular}

displaying the Point Sets A, B, C, and D were $\mathrm{y}=.65 \mathrm{x}+.65, \mathrm{y}=.84 \mathrm{x}+.25, \mathrm{y}=.84 \mathrm{x}+.54$, and $y=.81 x+.92$, respectively. To determine whether the judgments for these four sets of points differed from one another, judgments for each point set were collapsed over fixation-speed/wheel-speed discrepancies, and the resulting means were tested for differences. Point Sets A, B, C, and D had means of $2.32,2.38,2.68$, and 3.00 , respectively. One-tailed $t$ tests for correlated means showed that there was no difference between mean rating for Sets $A$ and $B$ $[\mathrm{t}(2)=.79, \mathrm{p}>.25]$. However, the difference between Sets $B$ and $C$ approached significance at the .05 level $[\mathrm{t}(2)=2.08, .05<\mathrm{p}<.1]$ and the difference between Sets $C$ and $D$ was significant $[t(2)$ $=3.38, p<.05]$.

Another comparison relevant to the vectorextrapolation hypothesis was made between the ratings given to the Fixate-Stationary Stimuli and those given to the $0 \%$ discrepancy trials of the SinglePoint Stimuli (i.e., Trials 4 and 8 ). In both sets of trials, the subjects' eyes were fixed on a stationary fixation point; these two sets of trials differed only in that the Fixate-Stationary Stimuli included a fixation point moving at $100 \%$ of the translatory velocity of the moving wheel. According to the vectorextrapolation approach, this additional fixation point should have caused the judged ratings to have been higher than in trials where it was absent. The average rating given to the Fixate-Stationary Stimuli was 1.74; for the Single-Point Stimuli, Trials 4 and 8 were given a combined rating of 1.52 . Although this difference was in the direction predicted by the vectorextrapolation hypothesis, a correlated $t$ test showed that the difference between these two means was not significant $[\mathrm{t}(6)=1.03, \mathrm{p}>.10]$. 


\section{EXPERIMENT 3}

In Experiments 1 and 2, ratings for the SinglePoint Stimuli, in which the subjects' eyes followed the moving fixation point, were compared to those for a control condition, the Fixate-Stationary Stimuli. The ratings for these Fixate-Stationary Stimuli were made in response to a single rim point moving in the presence of two fixation points: a "dummy" fixation point that traveled at $100 \%$ of the translatory velocity of the rolling wheel and a "true" fixation point that remained stationary. Since the vector-extrapolation hypothesis suggests that the addition of a stationary fixation point can affect the process of establishing a frame of reference, a more appropriate comparison, for the purposes of this investigation, is between ratings of displays that contain both a moving and a stationary fixation point. Under these circumstances, the number of points being displayed and the relative motions of these points will be identical in the two conditions that are being compared. To allow this, Experiment 3 included displays that contained two fixation points: one remained stationary and the other moved at some proportion of the translatory velocity of the moving wheel. Relevant data were obtained from judgments made to Single-Point Stimuli when the eyes fixated either the moving or the stationary fixation point. It was expected that, in accord with an eye-movement account, rated motions for the single-rim-point stimuli would be determined by how closely the pursuit eye movements, and not the fixation-point motion, matched the translatory velocity of the rolling wheel. It was also expected that the addition of a stationary fixation point would have little effect on judgments made to Single-Point Stimuli when the moving fixation point was being tracked.

To enable the appropriate comparisons to be made, Experiment 3 presented subjects with three sets of stimuli. All displayed the same eight Single-PointStimulus trials used in Experiments 1 and 2 (see Table 1). In two of these stimulus sets, however, a stationary fixation point was attached to the center of the screen, just above the path of the variablespeed fixation point. For one set of trials, termed the Fixate-Stationary Stimuli (as in Experiments 1 and 2), the subjects were instructed to keep their eyes on the stationary fixation point. For the other set of trials, termed the Fixate-Moving Stimuli, the subjects were instructed to follow the fixation point that moved at some proportion $(1.00, .67, .33$, or 0$)$ of the translatory velocity of the rolling wheel. A third set of trials, the Standard Single-Point Stimuli, presented just the eight Single-Point Stimuli and a variable-speed fixation point; no stationary fixation point was displayed. Thus, the Standard Single-Point Stimuli were exactly the same as what was termed the Single-Point Stimuli in Experiments 1 and 2.

\section{Method}

Apparatus and Procedure. The apparatus for Experiment 3 was identical to that used in Experiment 1. Experiment 3 required only some minor variations in the procedure that had been used in Experiment 1 in order to accommodate the new experimental trial types. As in Experiment 1, subjects in Experiment 3 received practice with the Single-Point Stimuli, displayed in the absence of a stationary fixation point. After the 32 practice trials (four repetitions of each of the eight trial types), stimuli for either the Fixate-Moving or the Standard Single-Point trials were displayed. The experimenter varied the order of presentation of these two sets of trials so that half the subjects saw the Standard SinglePoint Stimuli before the Fixate-Moving Stimuli, and half the subjects saw the two sets in the reverse order. For both sets of trials, subjects received four repetitions of each trial type. For the FixateMoving trials, the experimenter affixed a small white dot, measuring $.3 \mathrm{~cm}$ in diameter, to a point on the CRT's screen that was halfway along the path usually followed by the moving fixation point and approximately $.8 \mathrm{~cm}$ above this path. The subjects were told that the dot would serve as a fixation point later in the experiment, but that, for the present, they were to ignore it.

After the subjects had rated the Standard Single-Point and the Fixate-Moving Stimuli, the experimenter instructed the subjects to fixate on the stationary dot that had been ignored during the Fixate-Moving trials. The set of Fixate-Stationary trials that ensued consisted of one repetition of each of the eight trial types. Before being shown these Fixate-Stationary Stimuli, the subjects were informed that this last set of stimuli would consist of just eight trials.

Unlike Experiments 1 and 2, the experimenter remained in the experimental room with each subject for the duration of the experiment. The subjects were told that the experimenter, seated behind the CRT, was there to make sure the proper pursuit eye movements were executed. Except for giving the necessary instructions prior to the display of each of the three different sets of experimental trials, the experimenter remained silent.

Subjects. Six subjects, all having normal or corrected-to-normal vision, participated in this experiment. The subjects were recruited from the University of Oregon's Cognitive Laboratory subject pool, and received $\$ 2.50$ as compensation. The data from one subject who stated she was unable to perform the experiment, were discarded.

\section{Results}

Responses given to the Standard Single-Point Stimuli were no different from those given to the Fixate-Moving Stimuli for data collapsed over the four fixation-speed/wheel-speed discrepancies $[F(1,4)<1]$. Table 4 lists the data for the Standard Single-Point and the Fixate-Moving Stimuli in Columns 3-4 and 5-6, respectively. The linear trend for both sets of stimuli combined was significant $[F(1,4)=167.46, p<.01]$. Regression lines for the data from the Standard Single-Point and FixateMoving Stimuli had the form $y=.71 x+.56$ and $y=.66 x+.68$, respectively. From these analyses it appears that the presence of the extra stationary fixation point in the Fixate-Moving Stimuli had no effect on responses given to the Single-Point Stimuli.

To determine whether the moving fixation point affected perception of the Single-Point Stimuli when the eyes remained fixed on the stationary fixation point, data for the Fixate-Stationary Stimuli were analyzed for linear trend. The trend analysis for the Fixate-Stationary data (see Table 4, Columns 7-8) 
Table 4

Responses for the Standard Single-Point Stimuli, Fixate-Moving and Fixate-Stationary Stimuli, Experiment 3

\begin{tabular}{|c|c|c|c|c|c|c|c|}
\hline \multirow[b]{2}{*}{$\begin{array}{c}\text { Trial } \\
\text { Numbers }\end{array}$} & \multirow[b]{2}{*}{$\begin{array}{l}\text { Fixation Speed/ } \\
\text { Wheel Speed }\end{array}$} & \multicolumn{2}{|c|}{ Standard Single-Point } & \multicolumn{2}{|c|}{ Fixate-Moving } & \multicolumn{2}{|c|}{ Fixate-Stationary } \\
\hline & & $\begin{array}{c}\text { Mean } \\
\text { Responses }\end{array}$ & $\sigma^{2}$ & $\begin{array}{c}\text { Mean } \\
\text { Responses }\end{array}$ & $\sigma^{2}$ & $\begin{array}{c}\text { Mean } \\
\text { Responses }\end{array}$ & $\sigma^{2}$ \\
\hline $\begin{array}{l}1,5 \\
2,6 \\
3,7 \\
4,8\end{array}$ & $\begin{array}{r}1.00 \\
.67 \\
.33 \\
0.00\end{array}$ & $\begin{array}{l}3.34 \\
2.75 \\
2.13 \\
1.30\end{array}$ & $\begin{array}{l}.77 \\
.67 \\
.54 \\
.51\end{array}$ & $\begin{array}{l}3.25 \\
2.68 \\
2.13 \\
1.22\end{array}$ & $\begin{array}{l}.98 \\
.40 \\
.54 \\
.34\end{array}$ & $\begin{array}{l}1.60 \\
1.80 \\
1.70 \\
1.50\end{array}$ & $\begin{array}{l}.55 \\
.95 \\
.76 \\
.56\end{array}$ \\
\hline
\end{tabular}

was not significant $[F(1,4)<1]$. A regression line fitted to these data had the form $y=.04 x+1.55$.

That pursuit eye movements themselves, and not the mere presence of a moving fixation point, strongly affected the perceived motion of the rim points, can be seen by comparing the slopes of the regression lines for the response data of the Fixate-Moving and the Fixate-Stationary Stimuli. The slope of the FixateStationary regression line was .04; thus, almost no change in the perceived pattern of the single-rimpoint's motion occurred when the ignored (i.e., the "dummy") fixation point increased its velocity relative to that of the, rolling wheel. However, for the FixateMoving Stimuli, in which the eyes pursued the moving fixation point (while a stationary fixation point functioned as the "dummy" fixation point), the slope of the regression line was .66. This shows that as the velocity of the fixation point relative to that of the wheel went from $0 \%$ to $33 \%$ to $67 \%$ and then to $100 \%$, the pattern traced out by the rim point was judged to become more circular by about .66 units for each of these four decreases in fixation-pointspeed/wheel-speed discrepancy. Thus, it took pursuit eye movements, not just the presence of a moving fixation point, to bring about changes in perception. Also in accord with the argument being made here was the finding that the interaction between the linear trends of the data for the Fixate-Moving and the Fixate-Stationary Stimuli was significant $[F(1,4)$ $=284.59$.

If eye movements alone accounted for the perceptions of the motion of single-point patterns used here, there should have been no difference between ratings given Trials 4 and 8 combined of the Standard Single-Point Stimuli, Trials 4 and 8 combined of the Fixate-Moving Stimuli, and all trials of the FixateStationary Stimuli (see Table 4): in all three cases, a single rim point was being observed with the eyes held stationary. However, correlated $t$ tests showed that the average responses given by subjects to all Fixate-Stationary trials $(1.65)$ were significantly higher than those given to Trials 4 and 8 of the Standard Single-Point Stimuli (1.30) and to Trials 4 and 8 of the Fixate-Moving Stimuli (1.22) $[\mathrm{t}(4)=$ 5.81 and 13.64 , respectively, both ps $<.01$, two-tailed test]. It is possible that the higher responses given all trials of the Fixate-Stationary Stimuli might have been partly due to a response bias. Since the FixateStationary trials were presented last in the experiment as a block of eight trials, subjects might, due to their previous experience of responding to a proportion of each set of trials using high-numbered ratings, have become increasingly biased towards making higher ratings as the Fixate-Stationary trials continued. To examine this possibility, average ratings for the first four Fixate-Stationary trials (mean rating $=1.45$, $\sigma^{2}=.04$ ) were compared to average ratings for the last four Fixate-Stationary trials (mean rating $=1.85$, $\left.\sigma^{2}=.14\right)$. A correlated $t$ test showed that these means were significantly different $[\mathrm{t}(4)=2.36$, $\mathrm{p}<.05]$. Thus, a response bias may have raised the average ratings given to the Fixate-Stationary Stimuli. A randomization procedure which allowed FixateStationary trials to be interspersed among FixateMoving trials, a procedure prevented in this experiment by a hardware limitation, could probably have reduced this effect.

\section{DISCUSSION}

The hypothesis being investigated here-that pursuit eye movements can establish a frame of reference for the analysis of motion-was clearly supported by both the analysis of trend of the judgments for the Single-Point Stimuli and by the lowering of the overall "error" score when unsatisfactory eye-movement trials were excluded from subjects' data. The trend analysis indicated that as the discrepancy between the speed of the pursuit eye movements and the speed of the hub of the rolling wheel was decreased, the pattern that was perceived to be traced out by the point on the rolling wheel's rim was judged to be increasingly circular. The error score, which was derived from squaring the difference between actual and predicted judgments, was statistically less satisfactory than the trend analysis for supporting the pursuit-reference-frame hypothesis, since it did not quite reach significance at the .05 level. However, this error data did indicate that the average ratings given to the stimuli agreed more closely with the predicted values when ratings based on unsatisfactory pursuit eye movements were excluded. 
That the ratings given to the experimental stimuli were due to pursuit eye movements and not to the presence of the fixation point was supported by the finding that when the eyes remained stationary, a fixation point moving at some proportion of the translatory velocity of the rolling wheel had no effect on the ratings given to Single-Point Stimuli. That is, if the eyes remained stationary, the perceived motion of a single lighted point on the rim of a rolling wheel was independent of the relative velocities of the "dummy" fixation point and the rolling wheel. Furthermore, as predicted by the pursuit-eye-movement hypothesis, ratings given to all trials viewed with the eye held stationary indicated that cycloidal rather than circular motion had been perceived.

Although the parameters of the best-fit lines for the judgments of the Single-Point Stimuli used in Experiments 1,2 , and $3(y=.60 x+.83$ for Experiments 1 and 2 combined, and $y=.71 x+.56$ for the Standard-Single-Point trials of Experiment 3) generally agreed with the predicted values $(y=x+1)$, it is likely that a closer match could have been achieved by exerting a more precise control over pursuit eye movements. In the procedures used in these experiments, subjects were simply asked to pursue the fixation point; even in Experiment 2, where judgments based on unsatisfactory eye movements were discarded, only obvious violations of the pursuit-eyemovement instructions could be detected.

In addition to demonstrating that pursuit eye movements can play a role in establishing frames of reference, the data from this experiment also indicated that a vector-abstraction process may have occurred under some of the experimental conditions used here. The data relevant to this phenomenon showed that, under identical pursuit-eye-movement conditions, a pattern was judged to be increasingly circular as the number of points having motion components in common with that of the hub of the rolling wheel was increased. This was evident from judgments given to the Multipoint Stimuli (see Table 3). The regression lines which were fitted to Stimulus Sets A, B, C, and D showed that as the number of rim points was increased, subjects judged the patterns traced out to be increasingly circular. For Stimulus Sets B, C, and D, the regrssion lines had the form $\mathrm{y}=.84 \mathrm{x}+.25, \mathrm{y}=.84 \mathrm{x}+.54$, and $\mathrm{y}=.81 \mathrm{x}+.92$, respectively. The $y$ intercepts of these equations show that, at all fixation-speed/wheel-speed discrepancies, judgments made by subjects became more circular (by about .3 units) for each additional point that was displayed on the wheel's rim. As mentioned previously, however, pursuit eye movements could not be closely monitored in this study; thus, the small variations in the perceived motions of the different sets of Multipoint Stimuli may have resulted from differences in pursuit eye movements rather than a vector-extrapolation process.

In sum, these experiments demonstrated clearly that, as Stoper (1973) has suggested, frames of reference can be established by pursuit eye movements. If a vector-extrapolation process played any role in establishing frames of reference for the stimuli employed here, its effect was only minor.

\section{REFERENCES}

Hochberg, J., \& Fallon, P. Perceptual analysis of moving patterns. Science, 1976, 194, 1081-1083.

Johansson, G. Configurations in event perception: An experimental study. Uppsala: Almqvist \& Wicksell, 1950.

STOPER, A. E. Apparent motion of stimuli presented stroboscopically during pursuit movement of the eye. Perception \& Psychophysics, 1973, 13, 201-211.

Wallach, H. Perception of motion. Scientific American, 1959 , 201(1), 56-60.

(Received for publication March 22, 1978; revision accepted September 26, 1978.) 